Aus der Universitätsklinik und Poliklinik für Hautkrankheiten in Würzburg. (Vorstand: Prof. Dr. K. Zieler.)

\title{
Weitere Erfahrungen mit Salvarsan.
}

Von Dr. Artur Schmitt, Assistent der Poliklinik.

Seit mehr als $1 \frac{1}{2}$ Jahren wenden wir zur Salvarsaneinverleibung vorwiegend die intravenöse Methode an. Das Krankenmaterial, das wir auf diese Weise behandelt haben, setzt sich folgendermaßen zusammen:

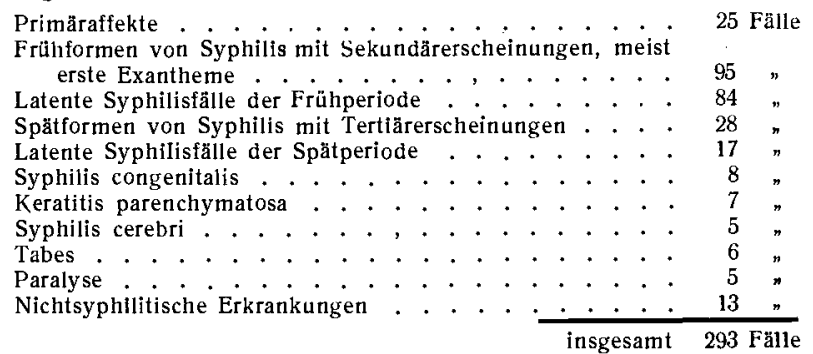

Bei diesen 293 Fällen wurden zusammen 448 intravenöse Injektionen gemacht; bei 40 Fällen wurde daneben Salvarsan subkutan (nur vereinzelt) oder intramuskulär - meist das Schindle rsehe Joha - irjiziert, bei 38 Fällen gleichzeitig oder anschließend eine Quecksilberbehandlung - meist mit Oleum cinereum - eingeleitet. In allen Fällen, bei denen die Kombination unterblieb, geschah dies zwecks Sammlung klinischer Erfahrungen oder aus äußeren Gründen.

Wir verwenden von jeher die alkalische Lösung nach der Vorschrift Schreibers und injizieren auch heute noch mit der Schreiberschen Spritze. Nur in letzter Zeit wurde infundiert (Weintraud). Wir sind mit diesen beiden Instrumentarien völlig ausgekommen und haben nie das Bedürfnis empfunden, eines der vielen empfohlenen ,,verbesserten Instrumentarien" zu benutzen.

In der Dosierung richten wir uns nach dem Untersuchungsergebnis der inneren Organe und nach dem Allgemeinzustand des Kranken. Bei Männern geben wir im allgemeinen $0,4-0,6$, bei Frauen $0,3-0,5$, bei Kindern je nach dem Lebensalter entsprechend weniger. Vorsichtig sind wir in der Dosierung bei Herz-, Nieren- und Lungenerkrankungen, besonders nach unseren früheren Erfahrungen bei florider Tuberkulose; ferner bei Affektionen des Gehirns und Rückenmarks, mochten sie nun spezifischer oder nichtspezifischer Natur sein, was ja zuvor oft nicht eindeutig zu entscheiden ist. Auch bei älteren Leuten (60-72 Jahre) haben wir Dosen von $0,3-0,4$ ohne jeden Schaden angewendet. Wir glauben deshalb nicht (wie Polland u. a.), dab etwaige Nebenerscheinungen durch das hohe Alter bedingt werden.

Unsere therapeutischen Erfolge bei floriden primären, sekundären und tertiären Erscheinungen der Syphilis decken sich vollständig mit den guten, nicht übertriebenen Resultaten anderer Autoren und stehen im Einklang mit der bekannten Wirkung des Quecksilbers auf die einzelnen Formen der syphilitischen Manifestationen, nur daß der symptomatische Erfolg des Salvarsans bedeutend rascher eintritt. Einen gegen Salvarsan refraktären Fall haben wir nicht zu Gesicht bekommen; dahingegen erwies sich der therapeutische Wert der intravenösen Salvarsaneinverleibung in einem Falle, welcher auf allerdings ungenügende Quecksilberdosen nur äußerst unvollständig reagierte.

H. O. Tuberoserpiginöses Syphilid am linken Mundwinkel, seit über einem Jahre bestehend, von über Fünfmarkstïckgröße. Zwei Quecksilberkuren (Asurol und Oleum cinereum, Kalomel, dies allerdings nur in mäßigen Dosen und mit zu langen Pausen (Reisender), und Jodkali hatten nur anfangs Erfolg. Röntgentherapie (volle Erythemdosen) verursachte nur ein teilweises Finfallen der derben, ziemlich stark 
prominentelı Wucherungen. Behandlung mit Kohlensäureschnee von negativem Erfolg. Zwei Johainjektionen à 0,4 Salvarsan von nur bescheidenem Einfluß. Endlich entschloß sich der Kranke zur intravenösen Salvarsaninjektiou, welche $\mathrm{ihm}$ schon längst angeraten war. 26. Juli 1911 0,5 Salvarsan intravenös. Rapider und vollständiger Rückgang. Kein Rezidiv seit neun Monaten.

Auf der anderen Seite sahen wir auch unter Salvarsan Primäraffekte sehr langsam zurückgehen.

P. G. Sechs Wochen alte Primäraffekte (2). WaR. 0. Spirochäten $\dagger$. Innerhalb 14 Tagen 1,5 Salvarsan intravenös und vierma $0,1 \mathrm{Hg}$ salizvl, dann $\mathrm{Hg}$-Pflaster und vier Injektionelı Ol. eill. $(0,06$; 0,$08 ; 0,1 ; 0,1 ; \mathrm{Hg}$ ) in dell folgenden fünf Wochen. Beide Prinäraffekte noch deutlich infiltriert!

Unsere sechs Fälle von Keratitis parcnchymatosa dürfen wegen der durchwegs positiven Wa.R. mit größter Wahrscheinlichkeit als auf syphilitischer Grundlage beruhend angesehen werden. Sie standen mit Ausnahme eines Falles unter Beobachtung der Universitätsaugenklinik, zeigten aber nach allerdings nur einmaliger Injektion - keine für das Mitte in Anspruch zu nehmende Beeinflussung. In einem anderen Falle ist nach Mitteilung des mitbehandelnden Augenarztes nach zweimaliger intramuskulärer Injektion von trüb alkalischer Lösung eine bedeutende und schnelle Besserung des Sehvermögens eingetreten, wovon wir uns selbst überzeugen koninten.

Der Fall ist früher eingehend besprochen worden. ${ }^{1}$ )

In zwei Fällen von Iritis papulosa war der momentane Erfolg ausgezeichnet; der eine Fall allerdings leidet jetzt nach $3 / 4$ Jahr an einem Rezidiv.

Ein Fall progressiver Opticusatrophie wurde durch vier intravenöse Injektionen zum Stillstand gebracht und ist jetzt neun Monate nach der letzten Injektion noch stationär. Das Sehvermögen wurde nicht beeinflußt, nur die fortschreitende Gesichtsfeldeinengung machte keine weiteren Fortschritte.

Von den fünf Fällen von Syphilis cerebri war in zwei Fällen der Erfolg der Behandlung recht gut; in dem dritten Falle jedoch trat nach Salvarsan und Oleum cinereum nur eine vorübergehende, nicht progrediente Besserung ein während erst eine energische Kalomelkur die Erscheinungen wesentlich beeinflußte. Der vierte Fall (nur eine Injektion) zeigte nur geringe Besserung, eine Hemiplegie (seit vier Monaten) keine wesentliche Veränderung trotz vier Injektionen im Verlauf von fünf Wochen.

Die im allgemeinen negativen Frfolge bei Tabes und Paralyse entsprechen den geringen Erwartungen, welche wir auf Grund der pathologischen Veränderungen an die Therapie geknüpft haben. Was wir beobachteten, waren vorläufige oder vorübergehende Besserungen der Erscheinungen.

So in einem Falle von Tabes incipiens Verschwinden der Blasen störungen, in einem vorgeschrittenen Fall Besserung dieser Beschwerden in einem weiteren Falle günstige Beeinflussung der Darmkrisen. Dieser Fall war aber schon vorher unter $\mathrm{Hg}$ gebessert; die Wa.R. ist seit $11 / 2$ Jahren negativ geblieben. In einem Falle von Paralyse im Anfangsstadium trat nach den einzelnen Injektionen vorübergehende Besserung ein, in der Zwischenzeit aber hat sich der Fall zu einer ausgesprochenen Paralyse entwickelt. Zwei andere Fälle wurden deutlich verschlechtert direkt im Anschluß an die Einspritzung.

Während wir also bei progressiver Paralyse nie einen Erfolg gesehen haben, glauben wir, daß bei Tabes eine Besserung, mindestens ein Stillstand $\mathrm{zu}$ erreichen ist, und würden hierfür entschieden Salvarsan bzw. Salvarsan und $\mathrm{Hg}$ empfehlen.

Zwei Fälle von Leukoplakie, der eine ohne sicher nachweisbare syphilitische Grundlage wurde mit gutem, aber unvollständigem Erfolge behandelt, der andere (früher sichere Syphilis) bei nur kurzer Beobachtung ohne deutlichen Erfolg.

Ein Fall von wahrscheinlicher Hodgkinscher Krank heit (Lymphomatosis granulomatosa) und sicherer Syphilis (mikropapulöses Syphilid) zeigte bereits nach einer Injektion eine günstige Beeinflussung der Drüsentumoren und des Blutbildes bei schneller Abheilung des Syphilids; jedoch nur eine geringe Wirkung auf das Allgemeinbefinden.

1) Zi eler, Würzburger Aerzteabend, 23. Mai 1911; Münchener medizinische Wochenschrift 1911 , No. 26.
Ein Fall von Lymphogranulomatose (?) nicht sicherer Aetiologie (Tuberkulose oder Syphilis? mikroskopische Untersuchung wurde verweigert) (eine Injektion) blieb unbeeinflußt.

Von Fällen nicht syphilitischer Erkrankungen, die mit Salvarsan behandelt wurden, seien erwähnt:

Ein Fall von Pemphigus chronicus (benignus?) mit nur scheinbarer Besserung nach drei Injektionen.

Ein Fall von Lichen ruber planus ohne Frfolg (eine Injektion), während später Arsazetin die Heilung herbeiführte.

Ein Fall von sogenanntem Lupus pernio, ohne Beeinflussung durch je eine intramuskuläre und intravenöse Injektion. ${ }^{1}{ }^{1}$

Ein Fall von Polyzythä mie (eine Injektion) ohne Beeinflussung.

Ein Fall von inoperablen Karzinomuetastasen an den prä- und postaurikularen Lymıhdrïselı - Operation verweigert - mit dem Erfolg, daßs nach den beiden Injektionen nach anfänglichem Prickeln und Bohren in den Tumoren zunächst die Schmerzen verschwanden und der Tumoreninhalt zur teilweisen Verflüssigung kall; das Weiterwuchern der Geschwulst wurde jedoch nicht verhindert.

Ein weiterer Fall vou Karzino in der Muldschleimhaut (harter Gaumen), welcher unbeeinflußt blieb.

Ein Fall von mehrulals ohne Dauererfolg operiertem Ly nıpo. sarkom, das nach anfänglicher scheinbarer Besserung trotz zweier Injektionell rapid weiterwuchs.

Ein Fall von inoperablem Sarkom der rechten Halsseite unterhalb des Ohres, das bei zweimaliger Injektion eine Herdreaktion zeigte, indem unter Schwellung die Schmerzen in der Geschwulst stärker wurden, vier 'Tage nach der ersten Injektion reichlich blutig-schwärzliche Massen uuter Abnahme der Geschwulst ausgestoßen wurden, am Tage nach der zweiteu Injektion die Sekretausscheidung aus der Inzisionsöffnung reichlicher wurde. Zwei Monate später kam der Kranke dureh Kachexie ad mortem. ${ }^{2}$ )

Die Dauerwirkung des Salvarsans ist gerade bei intravenöser Einverleibung und bei unkombinierter Behandluing mit Ausnahme vereinzelter Fälle einer sehr früh einsetzenden Therapie nach unseren Erfahrungen keine nachhaltige. Wir beobachteten in einem Falle bereits vier Wochen nach der ersten Injektion von 0,6 Salvarsan Rezidive an den Tonsillen, welche nach erneuter Injektion rasch schwanden. Auch in anderen unkombiniert behandelten Fällen, soweit wir sie nicht ganz aus den Augen verloren, sondern diese sich nur zeitweise unserer Beobachtung entzogen haben, waren nach $1 / 4-3 / 4$ Jahren Rezidive eingetreten. Ein Fall von Syphilis congenitalis (2.l Jahre alt), welcher wegen eines gummösen Ulcus der Tonsille 0,4 Salvarsan injiziert erhalten hatte, bekam drei Monate später eine Keratitis parenchymatosa, die seit etwa einer Woche deutlich war. Die Kranke erhielt am 23. Januar 1912 und am 12. Februar je 0,5 Salvarsan. Bis Ende März ist jedoch keine wesentliche Besserung eingetreten.

Unsere Erfahrungen bestätigen im allgemeinen nicht nur, daß man nur mit äußerster Zurückhaltung Erwartungen an die Dauerwirkung einzelner Salvarsaninjektionen stellen darf sondern sie beweisen auch, daß der Zeitraum eines halben Jahres, in welchem keine klinischen Erscheinungen aufgetreten sind, nicht zu höher gespannten Erwartungen berechtigt.

Für die Kranken, welche in unserer Beobachtung bleiben, kommt als wertvolles Unterstützungsmittel in unserem therapeutischen Vorgehen die Wa.R. in Betracht, welche in solchen Fällen selbstverständlich mit möglichst großer Regelmäßigkeit angestellt wird. Dabei ist jedoch der negative Ausfall der Reaktion nicht zu überschätzen. Nicht nur sahen wir bei Untersuchung des Blutes in achttägigen Intervallen Schwankungen der Reaktion zwischen negativem und wieder stark positiven Ausfall, sondern erlebten ebenso wie andere auch bei negativer Wa.R. Rezidive auf der Schleimhaut. Frst in solchen Fällen, bei welchen über längere Zeiträume hin die Seroreaktion sich stets negativ hält und der klinische Befund - Rückgang der Drüsen - damit in Einklang steht, halten wir uns für berechtigt, die Wa.R. zur prognostischen Verwertung heranzuziehen.

Bei vier Fällen von Primäraffekten, welche vier bis acht Wochen nach der Infektion in unsere Behandlung gekommen sind und innerhalb eines Zeitraumes von vier bis acht Wochen mit einer bis drei intravenösen, drei gleichzeitig mit Joha, einer

1) ef. Zieler, Würzburger Aerzteabend, 28. November 1911 , Münchener medizinische Wochenschrift 1912 , No. 1 .

2) betr. der beiden Sarkomfälle of. Zi eler, Würzburger Aerzteabend, 28. November I9II, Münchener medizinische Wochenschrift 1912, No. 1 . 
mit Oleum cinereum behandelt worden sind, traten in der Zwischenzeit von 1 bis nahezu 11/2 Jahren keine Sekundärerscheinungen auf und ist die Wa.R. bei den einzelnen Nachprïfungen negativ geblieben. Derartig günstige Resultate, bei denen doch immerhin an die Möglichkeit einer Dauerheilung zu denken ist, sind vielfach auch von anderen Autoren berichtet, soda $B$ wir uns darauf beschränken, als Typus eines solchen Falles den folgenden intramuskulär behandelten anzuführen.

M. A. Infektion vor vier Wochen, Primäraffekt, keine Inguinaldrüsen, Spirochätenbefund positiv, Wa.R. 0. 26. November 1910 0,5 Salvarsan intraglutäal, trüb alkalische Lösung nach Alt an vier verschiedenen Stellen deponiert. 17. Dezember 1910: Primäraffekt geheilt, Induration noch fühlbar; keine Drüsen. 13. Januar 1911: Kein Drüsenschwellung, noch eben fühlbare Verhärtung des Primäraffekts. Wa.R. Selbsthemmung. 28. Februar 1911: Kubitaldrüsen, linkseitige Inguinaldrüsen deutlicher als früher, sonst nichts. Wa.R. 0.30. Mär 1911: Rechte Kubitaldrüse über bohnengroß, sonst Status idem. Wa.R. 0 . 25. April 1911: Status idem, Wa.R. 0. 30. Januar 1912: Wa.R. 0 . 22. Februar 1912: Primäraffektnarbe noch fühlbar, etwas hart

In diesem Fall scheint also eine Abortivheilung mit intramuskulärer Injektion gelungen zu sein (Drüsen?). Ueberhaupt haben wir den Eindruck, da $B$ die intramuskuIäre Salvarsananwendung zweifellos günstige Wirkungen entfalten kann, trotz aller Nachteile, die mit $\mathrm{i}$ hr verbunden sind. Insbesondere möchten wir den günstigen Verlauf des nächsten Falles darauf zurückführen.

H. Infektion vor $11 / 2$ Jahren: Syphilis latens, starke Polyseleradenitis. Wa.R. positiv. Bisher sehr schlecht und unregelmäßig behandelt (zwei Hg-Kuren). 29. November 1910: 0,5 Salvarsan intravenös. 1. Dezember 1910: 0,7 trüb alkalische Lösung intraglutäal (an vier verschiedenen Stellen). 3. Januar 1911: Wa.R. 0. Drüsenpakete zurückgebildet. 6. März 1911: Wa.R. 0. 20. Juli 1911: Wa.R. 0. 9. November 1911: Wa.R. 0. Bisher keinerlei Erscheinungen aufgetreten

Anderseits sahen wir zwei Fälle primärer Syphilis, be welchen trotz zwei- bis fünfmaliger intravenöser Injektion von Salvarsan innerhalb von drei Wochen resp. sechs Wochen die Wa.R. nach vier resp. acht Monaten noch oder wieder positiv war, allerdings ohne daß in der Zwischenzeit klinische Erscheinungen aufgetreten wären. In solchen Fällen, in denen das Fehlen von klinischen Erscheinungen und der negative Ausfall der Reaktion an die Möglichkeit einer Heilung denken lassen, schlagen wir den Kranken die Provokation der Wa.R durch eine intravenöse Salvarsaninjektion (nach Genne rich) vor. Wir haben diese bis jetzt in zwei Fällen von Frühlatenz und in zwei Fällen von Spätlatenz mit stets negativem Ausfall der Reaktion ausgeführt. Als Beispiel unserer Untersuchungen führen wir folgenden Fall an.

J. W. Infektion Ende Dezember 1910. 19. Februar 1911: Exzision des Primäraffekts. 0,4 Salvarsan intravenös. Wa.R. positiv 21. Februar 1911: $1 \mathrm{ccm}$ Joha. 16. März 1911: Wa.R. ? 23. Märr 1911: 0,5 Salvarsan intravenös. 2. Mai 1911: Wa.R. 0. 21. Juli 1911: Wa.R. 0. 26. Februar 1912: In der Zwischenzeit keinerlei Erscheinungen aufgetreten. Keine Kubita!- und Inguinaldrüsenschwellung. Wa.R. 0. 13. März 1912: 0,5 Salvarsan intravenös. 14. März 1912: Wa.R. 0. 16. März 1912 Wa.R. 0. 18. März 1912: Wa.R. 0. 20. März 1912: Wa.R. 0. 23. März 1912: Wa.R. 0. 25. März 1912: Wa.R. 0.

Die prognostische Verwertbarkeit dieser Methode muß jedoch erst eine größere Reihe von Untersuchungen und langzeitiger Beobachtungen erbringen.

Von sogenannten Neurorezidiven beobachteten wir einen Fall, von einem weiteren Fall unseres Materials erfuhren wir durch Bericht.

Fr. N. Infektion vor 10-14 Wochen. Oedema indurativum lab. min. sin., sehr dicht stehendes großpapulöses Exanthem, das fast nur das Gesicht freiließ, Plaques muqueuses. Wa.R. positiv. 13. März 1911: 0,4 Salvarsan intravenös. Rascher Rückgang der Erscheinungen. 26. Mai 1911: Vor fünf bis sechs Tagen Erbrechen, Kopfschmerzen, rechts Ohrensausen, Schwindel. [Knochenleitung beiderseits gestört, rechts sehr stark, links wenig. Taubheit rechts, Mittelohr beiderseits intakt. Unmöglichkeit, allein zu gehen. Schwindel, der sich späte beim Rückgang besonders nach rechts bemerkbar macht.' 27. Mai 0,4 intravenös. Wa.R. 0. 30. Mai: Fazialisparese vollständig zurückgegangen. Es besteht noch leichter Schwindel und noch starke Hörstörung rechts, ${ }^{,}$die aber schon gebessert. Acusticus links frei. 16. Juni: Wa.R. 0; 0,4 intravenös. Bis 2. September außerdem fünf Injektionen Oleum ciner. $=0,335 \mathrm{Hg}$. 22. Januar 1912: Gehör gut, |zeitweilig Ohrensausen. Typische Plaques muqueuses. Wa.R. positiv. 0,5 Salvarsan intravenös, anschließend Oleum ciner.-Injektionen.
H. R. Infektion vor vier Monaten. Plaques muqueuses, Polyscleradenitis universalis, Papeln an den Händen. Wa.R. positiv. 4. Fe bruar 1911: 0,35 Salvarsan intravenös. Daun weiterer Behandlung ferngeblieben. Mai 1911 anderswo zwei Injektionen Salvarsan intravenös. Ende Juli Schwindel, völlige Taubheit des rechten Ohres, Ohrensausen; Stockung der Sprache, viertägige Lähmung der rechten Hand; Versagen der Magentätigkeit. Durch Einleiten einer Hg-Kur nach Bericht Besserung.

Diese beiden Fälle von Neurorezidiven machen in dem von uns behandelten und bis zu sechs Monaten und darüber beobachteten Material von 46 Fällen über $4 \%$ aus, wenn man die mit Salvarsan allein behandelten, hierher gehörigen 29 Fälle als Ausgangspunkt benutzt, nahezu $7 \%$ aus. Dabei muß freilich zugegeben werden, daß bei der kleinen Zahl des Beobachtungsmaterials die prozentuale Berechnung leicht zu Uebertreibungen führt, und mag ferner eingeräumt werden, daß ein Teil unserer Fälle nicht mehr zur Beobachtung kam, weil sich keine Symptome in der Zwischenzeit gezeigt haben. Der von uns ermittelte Prozentsatz stimmt jedenfalls ziemlich überein mitden Beobachtungen von Rille, Finger, Scholz, Jadassohn. Die Berechnung Benarios auf die Gesamtzahl der behandelten Fälle erscheint uns nicht zulässig und höchstens irreführend. Im einzelnen hat ja Finger zur Genüge darauf hingewiesen.

Durch spezifische Behandlung der Neurorezidive haben wir unseren Standpunkt über die Aetiologie dieser Erscheinungen bereits gekennzeichnet, daß es sich nach unserer Meinung um Syphiliserscheinungen an den Nerven bzw. an den Meningen infolge unzureichender Salvarsanbehandlung handelt. Aber immerhin sind wir der Ansicht, daß die Neurorezidive seit Einführung der Salvarsantherapie sich gehäuft haben. Wir sind ferner der Ansicht, daß sie zum Teil mit der ungenügenden $\mathrm{Be}-$ handlung, wie sie für die Mehrzahl der Fälle die ein- oder zweimalige Injektion von Salvarsan bildet, zusammenhängen; also ein Zeichen für die mangelhafte Heilwirkung seltener oder kleiner Salvarsandosen sind. Ob als zweiter Faktor eine neurotrope Eigenschaft des Mittels, welche den Grund zu einem Locus minoris resistentiae für die Spirochäteneinnistung abgibt, mitspielt, ist bis jetzt nicht erwiesen und auch wohl schwer zu entscheiden. Jedenfalls glauben wir den Neurorezidiven dadurch am besten vorzubeugen, daß wir den Salvarsaninjelktionen eine energische Quecksilberbehandlung folgen lassen, und wir haben seit Durchführung solcher kombinierten Behandlung (seit Jahresfrist) keine mehr beobachtet.

An Nebenerscheinungen, wie sie nach der Injektion auftreten, haben wir Kopfschmerzen, Benommenheit, Ohrensausen, Schwindelgefühl, Leibschmerzen, saures Aufstoßen, Brechreiz, Erbrechen, Durchfälle, Herzklopfen, Pulsbeschleunigung, Nierenschmerzen - ohne daß Albumen nachgewiesen werden konnte - in einem Falle spurenweise Albunenausscheidung, in einem anderen wesentlich verminderte Urinausscheidung; Schüttelfröste, Hitze- und Frostgefühle. Schweißausbruch; Brennen an den Fußsohlen, Taubheitsgefühle an den Fingerspitzen beobachtet. Diese Erscheinungen waren alle vorübergehender Natur und meist schon am nächsten Tage verschwunden.

Temperatursteigerungen über $38^{\circ}$ sahen wir in 24 Fällen, davon zweimal bis zur Höhe von $40^{\circ}$. Meist war die Temperatur am nächsten Morgen "wieder normal, nur in drei Fällen noch am zweiten Abend erhöht. Die Mehrzahl der Nebenerscheinungen, besonders die Temperatursteigerungen, traten in der Zeit auf, in welcher wir noch nicht ausdrücklichen Wert auf frisch destilliertes Wasser legten, und ereigneten sich in vie größerem Prozentsatz in der Privatpraxis als auf der klinischen Station. Wir erklären uns dies dadurch, daß das Wasser in der klinischen Apotheke infolge des raschen Verbrauches stets frischer destilliert war als das für die Privatpraxis aus einerStadtapotheke bezogene. Seitdem wir nur frisch destilliertes Wasser benützen, sind auch in der Privatpraxis bei stündlicher Messung die Temperaturerhöhungen fast durchwegs ausgeblieben. Meist folgte auf eine Temperaturerniedrigung von 2-4 Zehntelgraden in den ersten drei bis vier Stunden eine geringe Erhöhung meist um 3-5 Zehntelgrade über die Anfangstemperatur nach sieben bis zehn Stunden. Der Temperaturanstieg entsprach in der Regel der Rückkehr des kurz nach der Injektion erhöhten 
Blutdruckes zur gewohnten Höhe. Wir sehen somit unsere Erfahrungen als Bestätigung der Wechselmannschen Mitteilung an. Freilich werden auch jetzt noch in seltenen Fällen Nebenerscheinungen beobachtet; diese müssen jedoch auf andere Momente zurückgeführt werden. Das Freiwerden hypothetischer Endotoxine, wie Ehrlich u. a. annahmen, scheint uns nicht für alle Fälle zur Erklärung genügend, da wir bei frischen Exanthemen oft keinerlei Fieberreaktion eintreten sahen trotz stärkster Reaktion des Exanthems. Für viele Fälle mag das ja zutreffen, denn nach der ersten Injektion sind leichte Temperatursteigerungen am häufigsten beobachtet worden. In anderen Fällen jedoch sahen wir erst bei der zweiten, kurz der vorausgehenden folgenden Injektion kräftige Temperaturanstiege erfolgen. Die scheinbare Willkür, mit welcher diese Erscheinungen auftreten, beweist eben, daß wohl verschiedene Momente in Frage kommen. Manchmal, um dies zu erwähnen, sahen wir bei vorausgegangener Verstopfung nach einer reichlichen Defäkation die Temperatur steil zur Norm abfallen.

Trotz der manchmal ernsteren Erscheinungen, welche wir auch bei frisch destilliertem Wasser gelegentlich beobachtet haben ${ }^{1}$ ), haben wir unter vorsichtiger Dosierung fünf Fälle kompensierter Herzfehler und fünf Fälle von Lungentuberkulose ohne Schädigung der Kranken injiziert. Bei einem Falle von beiderseitigem Lungenspitzenkatarrh stieg die Temperatur abends auf 38,8 (0,3 Salvarsan), bei einem anderen klinisch ähnlichen auf 39,8 (0,5 Salvarsan). In beiden Fällen traten keine klinischen Erscheinungen von seiten der Lungen auf und war die Temperatur am nächsten Morgen zur Norm zurückgekehrt. Der letzte dieser beiden Fälle hatte zwei Monate zuvor auf eine Injektion von Joha mit einer deutlichen Reaktion der Lungenherde und mit blutigem Auswurf geantwortet. Ebenso haben wir nach intraglutäaler Injektion der neutralen Suspension zweimal geringfügige Hämoptoë beobachtet. ${ }^{2}$ )

Ein Fall von syphilitischer Nephritis und ein anderer von syphilitischem Ikterus wurden rasch gebessert.

Todesfälle haben wir weder in Zusammenhang, noch im Anschluß an die Salvarsanbehandlung erlebt, und die neuerdings häufig gemeldeten Todesfälle französischer Autoren sind uns bisher unverständlich.

Ziemlich häufig sahen wir innerhalb der ersten zwölf Stunden das Auftreten der Jarisch-Herxheimerschen Reaktion auch an Schleimhautplaques und Tertiärerscheinungen - öfters von einer Stärke, wie wir sie bei Quecksilber nie beobachtet haben. In zwei Fällen traten auch am Halse und vereinzelt im Gesicht bis dahin nicht sichtbare Roseolaeffloreszenzen von mehr urtikariellem Charakter, aber demselben Typus wie am Stamme und an den Extremitäten auf. Fine Bezielung zwischen der Größe der verabreichten Dosis und dem Eintreten und der Stärke des Phänomens war in einer zur Erklärung des kausalen Zusammenhanges verwertbaren Weise nicht zu entdecken.

Ferner beobachteten wir zwei masernähnliche Arsenexanthe me, ausgebreitet über Rumpf, Gesicht und Extremitäten; in dem einen Falle auch vereinzelte Effloreszenzen auf der Wangenschleimhaut. Sie traten beide am dritten Tage nach der Injektion ein unter Temperaturanstieg und Beeinträchtigung des Allgemeinbefindens.

In zwei Fällen endlich klagten die Kranken nach der Salvarsaneinspritzung über starke Schmerzhaftigkeit an den zuvor angelegten Oleum cinereum-Depots. Wir haben das bei Kranken ohne Infiltrat aber nie gesehen. Der eine Fall bekam am zweiten Abend 38,9 Temperatur, litt an Kopfschmerzen, Appetitlosigkeit und „Metallgeschmack“ im Munde. Auf Behandlung im Schwitzkasten hin gingen sämtliche Erscheinungen bis zum nächsten Morgen fast ganz zurück. In einem Falle von Stomatitis mercurialis nach Oleum cinereum, welche von der Durchbruehstelle des dritten unteren Molarzahnes ausging, trat trotz lokaler und allgemeiner Behandlung und bei teilweiser vorhergehender Besserung nach intravenöser Salvarsaninjektion eine Verschlechterung der Hg-Stomatitis ein. Es steht diese unsere Beobachtung im Gegensatz zu der Veröffentlichung von le Blaye, welcher in einem Fall nach Salvarsaneinverleibung

1) ef. Zieler, Würzburger Aerzteabend, 28. November 1911; Münchener medizinische Wochenschrift 1912, No. 11.

$\left.{ }^{2}\right)$ cf. Zi eler, Deutsche medizinische Wochenschirift 1910, No. 44. sofortigen Stillstand und nach zweiter Injektion endgültige Heilung einer merkuriellen Stomatitis beobachtet haben will. Es scheint also das Salvarsan ganz allgemein vorhandene entzündliche Erscheinungen zu verstärken. Wir haben wie andere das auch bei Tuberkulose, bei Tumoren, an $\mathrm{Hg}$ - Injektionsstellen gesehen und haben geradezu den Eindruck, daß Hg-Depots unter Salvarsanwirkung schneller einschmelzen. Wir vermögen deshalbauch die von Herxheimer und Altmann beschriebene örtliche Reaktion bei Lupus nicht als eine spezifische der auf Tuberkulin eintretenden vergleichbaren Reaktion anzusehen, zumal sie nach unseren Erfahrungen wesentlich früher auftritt; sondern glauben, daß es sich hier um eine gänzlich unspezifische Wirkung handelt, die nicht nur bei vorhandener Infektion verschiedener Art (vgl. Voerner), sondern auch bei sonstigen entzündlichen Verändarungen (Hg-Depots etc.) sowie bei Tumoren auftritt. Die bei Psoriasis, Lichen ruber etc. beobachteten Reaktionen sprechen in gleichem Sinne.

Unsere Erfahrungen mit Salvarsan führten uns zur Aufstellung folgenden Heilplanes. In Fällen frisch ak quirierter Syphilis und in solchen mit floriden Erscheinungen leiten wir die Behandlung mit einer oder mehreren intravenösen Salvarsaninjektionen ein. Wir beabsichtigen damit die Abtötung großer Spirochätenmengen und die Beseitigung schädlicher und ansteckungsfähiger Erscheinungen. Da wir aber an diese Behandlung nicht von vornherein die Erwartung einer Sterilisatio totalis knüpfen, schlie Ben wir der intravenösen, eventuell mit einer intramuskulären (Joha) kombinierten Salvarsaninjektion möglichst eine energische Quecksilberbehandlung an. Auf diese Weise hoffen wir noch vorhandene Spirochäten zu treffen oder aus versteckten Herden ausbrechende Spirochäten an einer Ueberschwemmung des Organismus zu verhindern. Die Wahl eines möglichst lange in seiner Wirkung anhaltenden Präparates (Oleum cinereum, eventuell Kalomel) scheint uns dabei von wesentlicher Bedeutung. Im weiteren Verlauf behandeln wir unter Kontrolle der Wa.R. chronisch intermittierend mit möglichst energischen Quecksilberkuren und dazwischen gelegten Salvarsaninjektionen, wobei natürlich die Behandlung dem Einzelfalle angepaßst werden muB. Eine Abschwächung der Hg-Kuren, etwa durch vorwiegende Verwendung innerlicher Mittel oder schwach wirkender Injektionspräparate, halten wir für verfehlt. Im Gegenteil glauben wir der Mehrzahl der Kranken am besten zu dienen, wenn wir möglichst kräftige $\mathrm{Hg}-\mathrm{Ku}$ ren (Oleum cinereum, Kalomel) d u rch ene rgische Salvarsandosen verstärken, wie das u. a. Neisser und Gennerich empfehlen. Dann wird am ehesten eine ,abortive" Syphilisbehandlung ermöglicht werden.

Auch die Syphilisfälle mit viszeralen Erkrankungen spezifischer oder unspezifischer Natur streben wir in den Bereich der Salvarsanbehandlung zu ziehen, indem wir klinischen Befund und vorsichtige Dosierung in Einklang zu bringen suchen. Dazu ist freilich nötig, die auf schlimme Erfahrungen begründeten Kontraindikationen und die möglichen Folgeerscheinungen der Salvarsaninjektion, wie sie in der Literatur reichlich beschrieben sind, zu kennen. An absolute Kontraindikationen glauben wir nicht. Wenigstens glauben wir bei genïgend vorsichtiger Dosierung jeden Fall mit Salvarsan behandeln zu können. 